\title{
ON THE REPRESENTATION OF A GROUP OF FINITE ORDER
} AS AN IRREDUCIBLE GROUP OF LINEAR SUBSTITU. TIONS AND THE DIRECT ESTABLISHMENT OF THE RELATIONS BETWEEN THE GROUP.CHARACTERISTICS

By W. Burnside.

[Receired February 28th, 1903.-Read March 12th, 1903.]

Ir is hardly too much to say that the methods by which new ideas are introduced and new results obtained in any branch of mathematical science are in general indirect. In fact the historical development of a subject rarely follows the lines of the logical presentment that can be given when a sufficient number of results and their connexion with each other have become familiar.

A case in point is the theory of group-characteristics and the allied subject of the representation of a group of finite order as a group of linear substitutions. In the remarkable series of memoirs, published in the Berliner Sitzungsberichte, in which Herr Frobenius developed this theory, the actual starting point is an algebraical theorem connected with permutable matrices; and the methods made use of in the subsequent discussion turn largely on the properties of matrices. In the paper on "GroupCharacteristics"* in which I have given an account of Herr Frobenius's theory the methods used, though quite distinct from his, are at least as indirect. They involve the consideration of a continuous group from whose properties those of the group-characteristics are deduced.

The present paper is an attempt to deal with the theory of the representation of a group of finite order as a group of linear substitutions and the properties of the group-characteristics from what may be described as a self-contained point of view. No considerations or ideas are introduced foreign to those involved in the conceptions of an abstract group of finite order and of a group of linear substitutions of finite order. Moreover, the theorems made use of are those which lie at the foundations of the subject. It will be seen that the order in which the various parts of the subject are considered differs materially from that in which they occur in Herr Frobenius's memoirs. First, the complete reducibility (defined below) of a group of linear substitutions of finite order is taken; secondly,

"Proc. Lond. Math. Soc., Vol. xxxirr., pp. 146-162. This paper will be referred to as "G.-C." 
the number of distinct irreducible representations ; thirdly, the composition of the irreducible representations ; and, lastly, the group-characteristics and their properties.

1. Let $S_{1}, S_{2}, \ldots, S_{i}, \ldots$ be the operations of an abstract group $G$, and let $s_{1}, s_{2}, \ldots, s_{i}, \ldots$ be a set of linear substitutions in $n$ variables such that, for every pair of suffixes $i$ and $j$, if $S_{i} S=S_{k}$, then $s_{i} s_{j}=s_{k}$. The set of linear substitutions then form a group $g$ which is isomorphic with $G$. This group of linear substitutions is said to constitute a representation of $G$, the substitution $s_{i}$ being the representative of the abstract operation $S_{i}$. One and the same group of linear substitutions may thus give two or more representations of a given abstract group.

Let $s_{1}^{\prime}, s_{2}^{\prime}, \ldots, s_{i}^{\prime}, \ldots$ be a second set of linear substitutions, in the same number $n$ of variables, which gives a representation of $G, s_{i}^{\prime}$ being the representative of $S_{i}$. If it is possible to find a linear substitution $t$ in the $n$ variables, such that, for each suffix $i, t^{-1} s_{i} t=s_{i}^{\prime}$, the two representations of $G$ given by the first and second sets of linear substitutions are said to be equivalent. If, on the other hand, no such substitution as $t$ exists, the representations are called distinct.

A group of linear substitutions in $n$ variables is called reducible when it is possible to tind $n^{\prime}(<u)$ linear functions of the variables which are transformed among themselves by every substitution of the group. I propose to call a group of linear substitutions completely reducible when it is possible to choose the variables in such a way that they fall into sets with the properties (i) that the variables of each set are transformed among themselves by every substitution of the group; and (ii) that the group of linear substitutions in each separate set is irreducible.

2. In a memoir to appear shortly in the Acta Mathematica I have dealt with the questions of the reducibility of a group of linear substitutions of finite order, and of the number of distinct representations of a group of finile order as an irreducible group of linear substitutions. T'he discussion is based solely (i) on the possibility of representing any group of finite order as a regular group of permutations, and (ii) on the existence of at least one invariant Hermitian form for any group of linear substitutions of finite order." These two theorems are fundamental in connexion with groups of finite order, and the results arrived at in the memoir referred to are deduced from them without introducing any extraneous considerations, and in particular without any reference to group-characteristics.

*Loewy, Comptes Reudiss, Vol. cxxim., pp. 168-171 (1896); and Moore, Math. Ann., Vol. L., pp. 213-219 (1898). 
These results are (i) that every group of linear substitutions of finite order is completely reducible,* and (ii) that the number of distinct representations of a group of finite order as an irreducible group of linear substitutions is equal to the number of sets of conjugate operations contained in the group.

I propose here, starting from these results, as acquired by direct and comparatively simple reasoning, to deduce by a further direct process the properties of the group-characteristics.

3. Let $G$ be any abstract group of finite order $n$ with $r$ sets of conjugate operations, and denote by $g_{1}, g_{2}, \ldots, g_{r}$ the $r$ distinct representations of $G$ as an irreducible group of linear substitutions. One of these, which will always be denoted by $g_{1}$, necessarily operates on a single variable and consists of the identical operation only. If $g$ is any representation of $G$ as a group of linear substitutions, the complete reducibility of $g$ will be represented by the equation

$$
g=\sum_{1}^{r} a_{i} g_{i}
$$

where the $a$ 's are positive integers or zeros; and the equation expresses that the variables in $g$ may be so chosen that they fall into sets; there being, for each $i, a_{i}$ sets each of which undergoes an irreducible group of linear substitutions equivalent to $g_{i}$.

If in each of the substitutions $s_{1}, s_{2}, \ldots, s_{i}, \ldots$ of $g_{i}$ every coefticient is replaced by its conjugate imaginary, a new set of substitutions $s_{1}^{\prime}, s_{2}^{\prime}, \ldots, s_{i}^{\prime}, \ldots$ arises for which $s_{i}^{\prime} s^{\prime}=s_{k}^{\prime}$ if $S_{i} S_{j}=S_{k}$. This new set of substitutions therefore constitute a group, which is obviously irreducible, and gives rise to an irreducible representation of $G$, which must be included among $g_{1}, g_{2}, \ldots, g_{r}$. If this representation is equivalent to $g_{i}$, then $g_{i}$ is called self-inverse. If it is distinct, it may be denoted by $g_{i}$, and then $g_{i}$ and $g_{i}$ are called inverse representations.

Since $g_{i}$ is a group of finite order, any one of its substitutions $s_{i}$ is of finite order. Now a linear substitution of finite order can always be transformed so that, if $m$ is the number of variables operated on, it takes the form $t$

$$
x_{1}^{\prime}=\omega_{1} x_{1}, \quad x_{2}^{\prime}=\omega_{2} x_{2}, \quad \ldots, \quad x_{m}^{\prime}=\omega_{m} x_{m},
$$

* Independently of the investigatious of Herr Frobenius, this result has been directly proved by Maschke, Math. Alm., Vol. Irr., pp. 363-368 (1899); and more recently, as the particular case of a more general theorem, by Loewy, American Transactions, Vol. Iv., pp. 44-64 (1903).

† Many proofs have been given of this theorem; but, in the present connexion, I may refer to one I have given (Mcssenger of Mathematics, Vol.'xxvir., pp. 111-114) which involves no reference to the characteristic determinant, and depends directly and solely on $s$ being of finite urder. 
where $\omega_{1}, \omega_{2}, \ldots, \omega_{m}$ are roots of unity. The $\omega$ 's are called the multipliers of the substitution, and their sum $\omega_{1}+\omega_{2}+\ldots+\omega_{m}$ is defined to be the characteristic of $S_{j}$ in the irreducible representation $g_{i}$. Any other substitution $s_{k}$ of $g_{i}$ may similarly be transformed to the form

$$
x_{1}^{\prime}=\varpi_{1} x_{1}, \quad x_{2}^{\prime}=\varpi_{2} x_{2}, \quad \ldots, \quad x_{m}^{\prime}=\varpi_{m} x_{m} .
$$

If $S_{j}$ and $S_{k}$ are conjugate operations of $G$, then $s$ and $s_{k}$ are conjugate substitutions of $g_{i}$; and therefore

$$
x_{1}^{\prime}=\omega_{1} x_{1}, \quad x_{2}^{\prime}=\omega_{2} x_{2}, \quad \ldots, \quad x_{m}^{\prime}=\omega_{m} x_{m}
$$

ean be transformed into

$$
x_{1}^{\prime}=\varpi_{1} x_{1}, \quad x_{2}^{\prime}=\varpi_{2} x_{2}, \quad \ldots, \quad x_{m}^{\prime}=\varpi_{m} x_{m} .
$$

It may be immediately verified that this is only possible when the two sets of roots of unity $\omega_{1}, \omega_{2}, \ldots, \omega_{m}$, and $\varpi_{1}, \varpi_{2}, \ldots, \varpi_{n}$ differ only in the sequence in which they are written. Hence two conjugate substitutions of $g_{i}$ have the same multipliers; and the characteristics of two conjugate operations of $G$ in $g_{i}$ are equal to each other.

To the $r$ conjugate sets of operations in $G$ there will therefore correspond $r$ characteristics in $g_{i}$ which may be denoted by $\chi_{1}^{i}, \chi_{2}^{i}, \ldots, \chi_{j}^{i}, \ldots, \chi_{r}^{i}$. Taking, as will be done throughout, the first conjugate set to be that which consists of the identical operation only, $\chi_{1}^{i}$ is simply the number of variables operated on by $g_{i}$, and, if $p$ is the order of any operation of the $j$-th set, $\chi_{j}^{i}$ is the sum of $\chi_{1}^{i} p$-th roots of unity. To each irreducible representation of $G$ there belongs such a set of characteristics, the characteristics in the set belonging to $g_{1}$ each being unity. Further, it follows at once from the definition of inverse representations that, if $g_{i}$ and $g_{i}$ are inverse representations, then $\chi_{j}^{i}$ and $\chi_{j}^{i^{i}}$ are conjugate imaginaries for each $j$; while, if $g_{i}$ is a self-inverse representation, $\chi_{j}^{i}$ is real for each $j$. So also, if the $j$-th and the $j^{\prime}$-th are inverse conjugaive seis (i.e., if $S$ belongs to one when $S^{-1}$ belongs to the other), then $\chi_{j}^{i}$ and $\chi_{j}^{i}$ are conjugate imaginaries (or are real and equal); while, if the $j$-th is a selî-inverse set, $\chi_{j}^{i}$ is real for each $i$.

If the two irredicikls rapresentations $g_{i}$ and $g_{j}$ are set $u p$ in two distinct sets of $\chi_{1}^{i}$ and $\chi_{1}^{j}$ variables, the $\chi_{1}^{i} \chi_{1}^{j}$ products of the variabies undergo a linear substitution for every operation of $G$. This group moy be represented by $g_{i} g_{j}$, and the equation which expresses its compiete reduction is

$$
g_{i} g_{j}=\sum_{1}^{r} d_{i j k} g_{k}
$$

This equation implies ("G.-C.," p. 161) that for the characteristics of each 
conjugate set the equation

holds.

$$
\chi_{s}^{i} \chi_{s}^{j}=\sum_{1}^{r} d_{i j k} \chi_{s}^{k}
$$

If $d_{i j 1}$ is not zero, the above equation implies that there are $d_{i j 1}$ invariant bilinear functions of the two sets of $\chi_{1}^{i}$ and $\chi_{1}^{j}$ variables. Denote these variables by $x_{1}, x_{2}, \ldots, x_{x_{1}^{i}}$ and $y_{1}, y_{2}, \ldots, y_{x_{1}^{i}}$, and let $\chi_{1}^{i}$ be not greater than $\chi_{1}^{j}$. Any bilinear function of the $x$ 's and $y$ 's can then be expressed in the form $x_{1} Y_{1}+x_{2} Y_{2}+\ldots+x_{x_{1}^{i}} Y_{x_{1}^{i}}$. If this is invariant when the $x$ 's and $y$ 's undergo simultaneously the corresponding substitutions of $g_{i}$ and $g_{j}$, then $Y_{1}, Y_{2}, \ldots, Y_{x_{1}^{i}}$ must be transformed among themselves by every operation of $g_{j}$. Since $g_{j}$ is irreducible, this is only possible if $\chi_{1}^{j}=\chi_{1}^{i}$; and when this condition is satisfied there can only be one such invariant function. In fact, if both

$$
x_{1} y_{1}+x_{2} y_{2}+\ldots+x_{x_{1}^{i}} y_{x_{1}^{i}} \text { and } x_{1} Y_{1}+x_{2} Y_{2}+\ldots+x_{x_{1}^{i}} Y_{x_{1}^{i}}
$$

were invariant, then $y_{1}^{\prime}=Y_{1}, y_{2}^{\prime}=Y_{2}, \ldots, y_{x_{1}^{i}}^{\prime}=Y_{x_{1}^{i}}$ would be permutable with every operation of $g_{j}$, which is impossible since $g_{j}$ is irreducible. When $g_{i}$ is assigned the corresponding substitutions of $g_{j}$ are completely defined by the condition that $x_{1} y_{1}+x_{2} y_{2}+\ldots+x_{x_{1}^{i}} y_{x_{1}^{i}}$ shall be invariant; so that corresponding to a given $i$ there is just one $j$ such that $c_{i j 1}=1$.

On the other hand, the theorem of the existence of an invariant Hermitian form for $g_{i}$ is equivalent to the assertion that $g_{i} g_{i}$ has an invariant bilinear form. Hence

$$
d_{i i^{\prime} 1}=1, \quad d_{i j 1}=0 \quad\left(j \neq i^{\prime}\right) .
$$

\section{Let now}

$$
x_{s}=\sum_{t} \alpha_{s t k_{i}} x_{t} \quad\left(s, t=1,2, \ldots, \chi_{1}^{i}\right)
$$

be the representative in $g_{i}$ of one of the operations of $G$ belonging to the $j$-th conjugate set, the $h_{j}$ different operations of the set being given by the values $1,2, \ldots, h_{j}$ of the suffix $a$. When transformed by any substitution of $g_{i}$, these $h_{j}$ substitutions are permuted among themselves. Hence the linear substitution

$$
x_{s}^{\prime}=\sum_{t} x_{t} \sum_{a} \alpha_{s t i_{a}}\left(s, t=1,2, \ldots, \chi_{1}^{i}\right)
$$

is permutable with every substitution of $g_{i}$. But, since $g_{i}$ is irreducible, the only substitutions permutable with every substitution of $g_{i}$ are those which multiply each variable by the same number. Hence

$$
\sum_{a} \alpha_{s t k_{a}}=0(s \neq t) \text { and } \sum_{a} \alpha_{s s i_{a}}=\lambda \text {. }
$$


But

and therefore

$$
\begin{aligned}
\sum_{s} a_{s k_{a}} & =\chi_{j}^{i}, \\
\lambda & =\frac{h_{j} \chi_{j}^{i}}{\chi_{1}^{i}} .
\end{aligned}
$$

Hence, if $C_{j}$ is the sum of the operations of $G$ which belong to the $j$-th conjugate set, then, in the representation of $G$ given by $g_{i}, C_{j}$ is represented by the substitution

$$
x_{s}^{\prime}=\frac{h_{j} \chi_{j}^{i}}{\chi_{1}^{i}} x_{s} \quad\left(s=1,2, \ldots, \chi_{1}^{i}\right) .
$$

Now the sums of the operations of $G$ which belong to the different conjugate sets satisfy the equations ("G.-C.," p. 148)

$$
C_{j} C_{k}=\sum_{s} c_{j k s} C_{s}
$$

where $c_{j k s}$ are positive constants (or zeros) which can be calculated from the multiplication table of the group. These relations, being in fact identities, must hold between the expressions which correspond to the C's in any concrete representation. But in the concrete representation here considered $C_{j} C_{k}$ is the substitution which multiplies each variable by $\frac{h_{\mathrm{j}} h_{k} \chi_{j}^{i} \chi_{k}^{i}}{\left(\chi_{i}^{i}\right)^{2}}$; while $\sum_{s} c_{j k s} C_{s}$ is the substitution which multiplies each variable by $\sum_{s} c_{j k s} \frac{h_{s} \chi_{s}^{i}}{\chi_{\mathrm{l}}^{i}}$. Hence, for all values of $i, j, k$,

$$
h_{j} h_{l i} \chi_{j}^{i} \chi_{i s}^{i}=\chi_{1}^{i} \sum_{s} c_{j k s} h_{s} \chi_{s}^{i}
$$

From this system of relations, and those given by $d_{i i^{\prime} 1}=1, d_{i j 1}=0$, the complete system may be deduced algebraically.

If each side of (i) is multiplied by $\chi_{l^{\prime}}^{l}$, while $c_{j l s} h_{s}$ is replaced by $c_{j s^{\prime}} h_{l^{\prime}}$, ("G.-C.," p. 149), it becomes

$$
h_{j} \chi_{i}^{i} h_{k} \chi_{k}^{i} \chi_{k^{\prime}}^{l}=\chi_{\mathrm{s}}^{i} \Sigma_{s} \chi_{s}^{i} c_{j s^{\prime} k^{\prime}} h_{k^{\prime}} \chi_{k^{\prime}}^{l}
$$

Summing each side with respect to $k$,

Hence

$$
h_{j} \chi^{i} \sum_{l i} h_{l_{i}} \chi_{i}^{i} \chi_{k^{\prime}}^{l}=\chi_{1}^{i} \sum_{s} \chi_{s}^{i} \frac{h_{j} h_{s^{\prime}} \chi_{\chi^{l}}^{l} \chi_{s}^{l}}{\chi_{1}^{l}}
$$

for all values of $i, l, j$.

In this put $l=1$. Then, since $\chi_{1}^{i}$ and $\chi_{j}^{i}$ cannot be equal for all values of $j$,

$$
\sum_{i} h_{k} \chi_{k}^{i}=0
$$

for all values of $i$ except unity. 
Now in the equation $\quad \chi_{k}^{i} \chi_{k}^{i^{i}}=1+\sum_{s}^{\prime} d_{i i^{\prime} s} \chi_{k}^{s}$,

$\Sigma^{\prime}$ extending to all values of $s$ except unity, multiply each side by $h_{k}$, and sum with respect to $k$. Then

$$
\sum_{l_{i}} h_{l_{i}} \chi_{k}^{i} \chi_{l_{i}}^{i^{\prime}}=\sum_{k} h_{l_{i}}+\sum_{s}^{\prime} d_{i i^{\prime} s} \sum_{k} h_{k} \chi_{k}^{s}=n .
$$

Similarly, from

$$
\chi_{i}^{i} \chi_{i}^{j}=\Sigma_{s}^{\prime} d_{i j s} \chi_{k}^{s}, \quad\left(j \neq i^{\prime}\right),
$$

it follows that

$$
\sum_{k} h_{l_{i}} \chi_{i}^{i} \chi_{i}^{j}=0 \quad\left(j \neq i^{\prime}\right) .
$$

The two sets of relations

$$
\begin{aligned}
& \sum_{k} h_{l_{i}} \chi_{i}^{i} \chi_{i}^{j}=n, \\
& \sum_{i} h_{k} \chi_{k}^{i} \chi_{i}^{j}=0 \quad\left(j \neq i^{\prime}\right)
\end{aligned}
$$

give the ratio of the first minors in the determinant of the $\chi$ 's; and thence the two remaining sets of relations

$$
\begin{aligned}
\sum_{i} \chi_{k}^{i} \chi_{l^{\prime}}^{i} & =\frac{n}{h_{l i}}, \\
\sum_{i} \chi_{i}^{i} \chi_{l}^{i} & =0 \quad\left(l \neq k^{\prime}\right)
\end{aligned}
$$

immediately follow. I'he relations (i), (ii), (iii), (iv), and (v) are, in fact, the relations which connect the group-charanteristics ("G..-C.,". pp. 151, 153). 